\title{
MEAN VARIANCE AND GOAL ACHIEVING PORTFOLIO FOR DISCRETE-TIME MARKET WITH CURRENTLY OBSERVABLE SOURCE OF CORRELATIONS
}

\author{
Nikolai Dokuchaev ${ }^{1,2}$
}

\begin{abstract}
The paper studies optimal portfolio selection for discrete time market models in meanvariance and goal achieving setting. The optimal strategies are obtained for models with an observed process that causes serial correlations of price changes. The optimal strategies are found to be myopic for the goal-achieving problem and quasi-myopic for the mean variance portfolio.
\end{abstract}

Mathematics Subject Classification. 91B16, 91B70.

Received July 11, 2008. Revised November 30, 2008.

Published online June 18, 2009.

\section{INTRODUCTION}

The paper investigates optimal portfolio selection for discrete time stochastic market models. These problems have been studied intensively in the literature (see, e.g., [9] and more recent works or [10,13,20,21]. The singleperiod version of this problem was set and solved by Markowitz [17] in mean-variance setting. Multi-period version appears to be more challenging. If the market model is complete, then the martingale method can be used (see, e.g., [20]). Unfortunately, a discrete time market model is complete only under very restrictive assumptions. For incomplete discrete time markets, the main tool is dynamic programming. It requires to calculate the optimal value function backward starting at terminal time using the probability distributions of future prices. In Markovian setting, this function satisfies an anticausal backward Bellman equation, usually with finite dimensional state space. The dynamic programming method was used to obtain the existing results in mean-variance multi-period setting. In particular, this method was used by Li and $\mathrm{Ng}$ [13] for a model with independent security returns. Schweizer [21] and Henrotte [10] studied more general market model with serial correlations for the problem of mean-variance optimal hedging of random claims. The strategies obtained in these papers require the future probability distributions of the prices. In addition, these strategies depend on the choice of the time horizon.

For some special discrete time market models, optimal strategies are known to be myopic, i.e., they don't use the knowledge of the future probability distributions of the market prices and don't require forecast of these prices (see, e.g., $[9,12,19])$. More precisely, the strategy for discrete time is myopic if, at time $t$, it depends only on the historical observations and on the future probability distributions of the prices at time $t+1$.

\footnotetext{
Keywords and phrases. Discrete time market, multi-period market, myopic strategies, serial correlation, optimal portfolio, mean variance portfolio, goal achieving.

1 Department of Mathematics, Trent University, Ontario, Canada. nikolaidokuchaev@trentu.ca

2 Current address: Department of Mathematics and Statistics, Curtin University of Technology, GPO Box U1987, Perth, Australia. N.Dokuchaev@curtin.edu.au
} 
In other words, it requires one step forward prediction only, and the optimal choice of the portfolio at time $t$ does not depend on the terminal time and on the probability distributions of the prices at times after $t+1$.

In fact, the most well-known myopic optimal strategies were introduced for continuous time markets [18]. For the power and log utility functions, the optimal strategies are myopic for continuous time models if the market parameters (the appreciation rates and the volatilities) are currently observable and their forecast is not required. For the case of non-observable parameters, the optimal strategies are found to be myopic for the $\log$ utility function only. The impact of the observability or non-observability was intensively studied in the literature (see some references in $[1,8]$ ).

Unfortunately, the optimal strategies are rarely myopic for discrete time market models. Hakansson [9] showed that the optimal strategy is not myopic for $U(x)=\sqrt{x}$ if returns have serial correlation and evolve as a Markov process. The optimal strategies found in [10,21] are also non-myopic; they require forecast of future distributions. Clearly, it is interesting to find new examples of optimal myopic strategies.

In the present paper, we study optimal portfolio strategies and their myopicness for a incomplete discrete time market; new examples of optimal myopic strategies are found.

We consider the goal-achieving problem of minimization $\mathbf{E} U\left(\widetilde{X}_{T}-\kappa\right)$ and, following $\operatorname{Li}$ and $\mathrm{Ng}$ [13], the multi-period mean-variance problem $\operatorname{Var} \widetilde{X}_{T} \rightarrow \min , \mathbf{E} \widetilde{X}_{T} \geq$ const. Here $\widetilde{X}_{T}$ is the discounted wealth, $\kappa>X_{0}$ is a given target value, $U$ is a function that describes investor preferences. We consider only $U(x)=|x|^{\delta}$, where $\delta>0$.

Similarly to Schweizer [21] and Henrotte [10], we consider the market model with serial correlations for the price changes. The novelty is that we introduce a model with an observable parameter process that causes the correlations. This model can be considered as an analog of the continuous time market models such that the appreciation rate can be observed directly, i.e., when the Merton's optimal strategies are myopic for power utility functions.

We use some alternative to the dynamic programming method. More precisely, we calculate the optimal strategies directly, using the approach from Dokuchaev [3,5], where optimal and myopic strategies were obtained in discrete time setting for power and log utilities. The present paper extends this approach on the goal achieving and mean-variance problems. Convenient and compact explicit formulas for optimal strategies are obtained. For the goal-achieving problem, these strategies appear to be myopic. In the mean variance setting, the optimal strategy appears to be non-myopic, but the required information about the future distributions is very limited: for a multi-stock market with $n$ stocks, for an arbitrarily large $n$, it is required to estimate a single scalar parameter $\alpha$ defined by (3.7). As a consequence, the corresponding strategy does not leave the efficient frontier even if this parameter is calculated with an error, or if the time horizon is changed (Cor. 4.1). In addition, we found that, under natural assumptions, the probability distributions for the distant future have little effect on the value of this parameter. This also reduces non-myopicness of the optimal strategy. It is why this strategy can be called quasi-myopic.

The strategies obtained here can be compared with the continuous time strategies from Hipp and Taksar [11], Lim and Zhou [15], and Lim [14].

It can be added that Schweizer [21], Li and $\mathrm{Ng}$ [13], and Henrotte [10] considered the optimality criterions that include the total wealth. We consider the case when the discounted wealth is presented in the optimality criterions.

\section{THE MARKET MODEL}

We are given a standard probability space $(\Omega, \mathcal{F}, \mathbf{P})$, where $\Omega$ is the set of all elementary events, $\mathcal{F}$ is a complete $\sigma$-algebra of events, and $\mathbf{P}$ is the probability measure.

We consider a model of a market consisting of the risk-free bond or bank account with price $B_{t}$ and the risky stocks with prices $S_{t, k}$, where $t=0,1,2, \ldots$, and $k=1, \ldots, n$. Here $n \geq 1$ is the number of stocks. The initial prices $S_{0, k}>0$ and $B_{0}>0$ are given non-random variables. 
We assume that

$$
\begin{aligned}
& S_{t, k}=\rho_{t} S_{t-1, k}\left(1+\xi_{t, k}\right), \quad k=1, \ldots, n \\
& B_{t}=\rho_{t} B_{t-1}, \quad t=1,2, \ldots
\end{aligned}
$$

Here $\xi_{t, k}$ and $\rho_{t}$ are random variables such that $\xi_{t, k}>-1$ and $\rho_{t} \geq 1$ for all $t, k$.

In this setting, the single period risk-free return is $\rho_{t}-1$, and the single period return for the $k$ th stock is $\rho_{t}-1+\rho_{t} \xi_{k, t}$.

Let us describe our main assumptions about the distributions of $\left(\rho_{t}, \xi_{t}\right)$, where $\xi_{t}=\left(\xi_{t, 1}, \ldots, \xi_{t, n}\right)$. For $\xi_{t}$ and other similar vectors we mean vector-columns, i.e., $\xi_{t}$ takes values in $\mathbf{R}^{n}$.

Let $\mathcal{T}=\{T\}$ be a set of possible terminal times; $\mathcal{T} \subseteq\{1,2,3, \ldots\}$.

We assume that the following condition is satisfied.

Condition 2.1. There exists a sequence $\left\{\theta_{t}\right\}_{t \geq 1}$ of random variables that take values in a metric space $\mathcal{Z}$ such that the following holds for all $T \in \mathcal{T}$ :

(i) The pairs $\left\{\left(\xi_{t}, \rho_{t}\right)\right\}_{t=1}^{T}$ are mutually conditionally independent given $\theta_{1}, \ldots, \theta_{T-1}$.

(ii) For any $t=2, \ldots, T-1$, the pair $\left(\xi_{t}, \rho_{t}\right)$ is independent from $\theta_{t}, \ldots, \theta_{T-1}$ conditionally given $\theta_{1}, \ldots, \theta_{t-1}$. In addition, the pair $\left(\xi_{1}, \rho_{1}\right)$ is independent from $\theta_{1}, \ldots, \theta_{T-1}$ unconditionally.

(iii) $\theta_{t}, S_{t}$, and $B_{t}$, are currently observable, i.e., the values $\left\{\left(\theta_{s}, S_{s}, B_{s}\right)\right\}_{s \leq t}$ are known at time $t$. (It follows that $\xi_{t}$ and $\rho_{t}$ are currently observable).

(iv) At any time $t=0, \ldots, T-1$, the conditional distributions of $\left(\xi_{t+1}, \rho_{t+1}\right)$ given $\left(\theta_{1}, \ldots, \theta_{t}\right)$ are known. (If $t=0$, then the unconditional distribution is known.)

In this model, $\left\{\theta_{t}\right\}$ defines mutual dependence of the pairs $\left\{\left(\xi_{t}, \rho_{t}\right)\right\}$ i.e, it is the source of serial correlations; we assume that this process is currently observable. The vector $\theta_{t}$ represents observable market data that affect the probability distributions of price movement. It can include, for instance, historical trade volume, economic indicators, price index, a systematic market risk factor, the beta coefficients in the CAPM model, etc.

In fact, the vector $\left(\theta_{1}, \ldots, \theta_{t}\right)$ together with the conditional distribution of $\left(\xi_{t+1}, \rho_{t+1}\right)$ given $\left(\theta_{1}, \ldots, \theta_{t}\right)$ describes information about $\xi_{t+1}$ available at time $t$.

Note that Condition 2.1(iv) can be actually relaxed (see Rem. 3.3 below).

Example 2.1. Condition 2.1(i)-(ii) is satisfied, for instance, if there exists a metric space $\mathcal{W}$ and measurable functions $f_{t}(\cdot): \mathcal{Z}^{k-1} \times \mathcal{W} \rightarrow \mathbf{R}^{n+1}$ such that $\left(\xi_{t}, \rho_{t}\right)=f_{t}\left(\theta_{1}, \ldots, \theta_{t-1}, w_{t}\right), t=1,2, \ldots$, where $w_{t}$ are mutually independent random variables that take values in $\mathcal{W}$ and are independent from $\left\{\theta_{t}\right\}$.

The notation a.s. means "almost surely", i.e., "with probability 1".

We assume also that the following condition is satisfied.

Condition 2.2. For all $T \in \mathcal{T}$,

(i) $\mathbf{E}\left\{\rho_{t}^{T} \xi_{t}^{T} \mid \theta_{1}, \ldots, \theta_{t-1}\right\}<+\infty$ a.s. for $t=1, \ldots, T, t \leq k$ (if $t=1$, then we mean the unconditional expectation); and

(ii) For any $t=1, \ldots, T$, the conditional covariance matrix for $\xi_{t}$ given $\theta_{1}, \ldots, \theta_{t-1}$ is non-degenerate, and

$$
\mathbf{P}\left(\xi_{t, k}>0 \mid \theta_{1}, \ldots, \theta_{t-1}\right)>0, \quad \mathbf{P}\left(\xi_{t, k}<0 \mid \theta_{1}, \ldots, \theta_{t-1}\right)>0 \quad \text { a.s. }
$$

(If $t=1$, then we mean again the unconditional probability.)

Note that Condition 2.2(ii) represents a weakened modification of nondegeneracy condition from Schweizer [21]. Let $S_{t}=\left(S_{t, 1}, \ldots, S_{t, n}\right)$ (we mean again a vector column).

Let $X_{0}=1$ be the initial wealth of an investor at time $t=0$, and let $X_{t}$ be the wealth at time $t \geq 0$. We set that

$$
X_{t}=\beta_{t} B_{t}+\gamma_{t}^{\top} S_{t}
$$


where $\beta_{t}$ is the quantity of the bond portfolio and where $\gamma_{t}=\left(\gamma_{t, 1}, \ldots, \gamma_{t, n}\right)$ is the quantity of the stock portfolio (a vector-column), $\gamma_{t}^{\top} S_{t}=\sum_{k} \gamma_{t, k} S_{t, k}$. The pair $\left(\beta_{t}, \gamma_{t}\right)$ describes the state of the bond-stocks securities portfolio at time $t$. We call the sequences of these pairs strategies.

We consider the problem of choosing an optimal strategy. Some constraints will be imposed on current operations in the market.

Let $\mathcal{F}_{t}$ be the filtration generated by the process $\left(S_{t}, B_{t}, \theta_{t}\right)$ that represents the flow of observable data.

Definition 2.1. A strategy $\left\{\left(\beta_{t}, \gamma_{t}\right)\right\}$ is said to be admissible if

(i) The process $\left(\beta_{t}, \gamma_{t}\right)$ is adapted to the filtration $\mathcal{F}_{t}$.

(ii) For all $t \geq 0$,

$$
\mathbf{E}\left\{\left|X_{t}\right| \theta_{1}, \ldots, \theta_{t}\right\}<+\infty \quad \text { a.s. }
$$

(iii) For all $t \geq 0$,

$$
X_{t+1}-X_{t}=\beta_{t}\left(B_{t+1}-B_{t}\right)+\gamma_{t}^{\top}\left(S_{t+1}-S_{t}\right) .
$$

Note that $\beta_{t}$ and $\gamma_{t}$ are defined by $\left\{\xi_{m}, \rho_{m}, \theta_{m}\right\}_{m=1}^{t}$.

For the trivial, risk-free, "keep-only-bonds" strategy, the portfolio contains only the bonds, $\gamma_{t} \equiv 0$, and the corresponding wealth is $X_{t} \equiv \beta_{0} B_{t} / B_{0} \equiv \prod_{m=1}^{t} \rho_{m}$.

Let $R_{t} \triangleq \prod_{m=1}^{t} \rho_{m}$.

Definition 2.2. The process $\widetilde{X}_{t} \triangleq R_{t}^{-1} X_{t}$ is called the discounted wealth.

Set $\widetilde{S}_{t} \triangleq R_{t}^{-1} S_{t}, t>1, \widetilde{S}_{0} \triangleq S_{0}$. Clearly, $\widetilde{S}_{t}=\widetilde{S}_{t-1}\left(1+\xi_{t}\right)$.

Proposition 2.1. Let $\left\{\left(X_{t}, \gamma_{t}\right)\right\}$ be a sequence, and let the sequence $\left\{\left(\beta_{t}, \gamma_{t}\right)\right\}$ be an admissible strategy, where $\beta_{t}=\left(X_{t}-\gamma_{t} X_{t}\right) B_{t}^{-1}$. Then the strategy $\left\{\left(\beta_{t}, \gamma_{t}\right)\right\}$ is self-financing if and only if the process $\widetilde{X}_{t}=R_{t}^{-1} \widetilde{X}_{t}$ evolves as

$$
\widetilde{X}_{t+1}-\widetilde{X}_{t}=\gamma_{t}^{\top}\left(\widetilde{S}_{t+1}-\widetilde{S}_{t}\right), \quad t \geq 0 .
$$

It follows from this proposition that the sequence $\left\{\gamma_{t}\right\}$ alone suffices to specify admissible strategy $\left\{\left(\beta_{t}, \gamma_{t}\right)\right\}$. For $t \geq 0$, let random $\mathcal{F}_{t}$-adapted sets $\Delta_{t} \subset \mathbf{R}^{n}$ be given such that $0 \in \Delta$ for all $t, \omega$.

In the following definition we consider the infinite time horizon.

Definition 2.3. Let $\bar{\Sigma}$ be the set of all sequences $\gamma=\left\{\gamma_{t}\right\}$ such that the corresponding strategy $\left\{\left(\beta_{t}, \gamma_{t}\right)\right\}$ is admissible and such that $\gamma_{t} \in \Delta_{t}$ a.s.

We shall call again a sequence $\gamma \cdot \in \bar{\Sigma}$ strategy.

The constraints in Definition 2.3 cover many models with restrictions on the portfolio selection. There are some examples:

(i) Let $\Delta_{t}=\left\{v \in \mathbf{R}^{n}: \operatorname{dim}(v) \leq m\right\}$, where $m$ is given, and where $\operatorname{dim}(v)$ denotes the total number of non-zero components of a vector $v \in \mathbf{R}^{n}$. Then these constraints describe optimal portfolio compression problem when portfolio cannot include more than $m$ different stocks at any time.

(ii) Let $\Delta_{t}=\left\{v=\left(v_{1}, \ldots, v_{n}\right) \in \mathbf{R}^{n}: v_{k} \in\left[a_{k}, b_{k}\right]\right\}$, where $-\infty \leq a_{k} \leq b_{k} \leq+\infty$ are given constants. Then these constraints describe the model with restrictions on the quantity of shares.

(iii) Let $\Delta_{t}=\left\{v=\left(v_{1}, \ldots, v_{n}\right) \in \mathbf{R}^{n}: S_{k} v_{k} \in\left[a_{k}, b_{k}\right]\right\}$, where $-\infty \leq a_{k} \leq b_{k} \leq+\infty$. Then these constraints describe the model with restrictions on the wealth invested in particular stocks.

(iv) Let $\Delta_{t}=\left\{v=\left(v_{1}, \ldots, v_{n}\right) \in \mathbf{R}^{n}: v_{1} \geq 0, \ldots, v_{k} \geq 0\right\}$. Then these constraints describe the model where the short sale is forbidden for the stocks $1, \ldots, k$.

(v) Let $\Delta_{t}$ be finite or countable. Then these constraints cover the model where stock shares are not divisible arbitrarily. 


\section{Goal achieving PROBLEM}

Let the initial wealth $X_{0}=1$ and $\delta \in(0,+\infty)$ be given. Let $U(x)=|x|^{\delta}$.

We assume that Conditions 2.1-2.2 are satisfied for all $T \in \mathcal{T}$.

Let $\kappa>X_{0}$ be given.

Consider the following optimization problem:

$$
\text { Minimize } \quad \mathbf{E}\left\{U\left(\widetilde{X}_{T}-\kappa\right) \mid \theta_{1}, \ldots, \theta_{T-1}\right\} \quad \forall T \in \mathcal{T} \text { with probability } 1 \text { over } \gamma \cdot \in \bar{\Sigma} \text {. }
$$

In this setting, $\kappa$ serves as the target value.

Remark 3.1. Note that the required minimization for all possible time horizons and with probability 1 looks unusual for stochastic control setting, since it can be rarely achieved. For the case of a fixed $T$, the problem of maximization with probability 1 is also non-trivial, since admissible strategies do not use at time $t$ the future values $\left\{\theta_{k}\right\}_{k>t}$. However, we found below the optimal strategy that solves problem (3.1).

\section{Additional definitions}

Let mapping $H_{t}(v)=H_{t}(v, \omega): \mathbf{R}^{n} \times \Omega \rightarrow \mathbf{R}$ be defined as

$$
H_{t}(v) \triangleq \mathbf{E}\left\{U\left(1+v^{\top} \xi_{t+1}\right) \mid \mathcal{F}_{t}\right\}
$$

and let the mapping $H_{0}: \mathbf{R} \rightarrow \mathbf{R} \cup\{-\infty\}$ be defined as $H_{0}(v) \triangleq \mathbf{E} U\left(1+v^{\top} \xi_{1}\right)$.

It is assumed that, in the definition of $H_{t}(v)$, we select a version of the conditional expectation being the so-called regular conditional expectation [7]. This selection ensures that $H_{t}(v)$ is $\mathcal{F}_{t}$-measurable for any $\mathcal{F}_{t}$-measurable random vector $v$.

\section{The optimal strategy for the goal achieving problem}

Theorem 3.1. Let the sequence of random sets $\left\{\Delta_{t}\right\}$ be conditionally independent from the sequence $\left\{\left(\xi_{t}, \rho_{t}\right)\right\}$ given $\left\{\theta_{t}\right\}$. Let the strategy $\left\{\left(\beta_{t}, \gamma_{t}\right)\right\}=\left\{\left(\beta_{t}, \gamma_{t, 1}, \ldots, \gamma_{t, n}\right)\right\}$ be such that $\gamma_{t} \in \Delta_{t}$ a.s., and

$$
\gamma_{t, k}=\mu_{t, k} \widetilde{Y}_{t} \widetilde{S}_{t, k}^{-1}, \quad \beta_{t}=\frac{X_{t}-\gamma_{t}^{\top} S_{t}}{B_{t}}, \quad t=0,1,2, \ldots
$$

where

$$
\widetilde{Y}_{t} \triangleq \widetilde{X}_{t}-\kappa
$$

$X_{t}$ is the corresponding wealth, $\widetilde{X}_{t}$ is the corresponding discounted wealth, and where the random vector $\mu_{t}=$ $\left(\mu_{t, 1}, \ldots, \mu_{t, n}\right)$ is $\mathcal{F}_{t}$-measurable and such that

$$
H_{t}\left(\mu_{t}\right) \leq H_{t}(v) \quad \forall v=\left(v_{1}, \ldots, v_{n}\right) \in \mathbf{R}^{n}:\left(v_{1} \widetilde{Y}_{t} \widetilde{S}_{t, 1}^{-1}, \ldots, v_{n} \widetilde{Y}_{t} \widetilde{S}_{t, n}^{-1}\right) \in \Delta_{t} .
$$

Then this strategy is admissible, i.e., $\gamma . \in \bar{\Sigma}$ with given $\kappa$, and it is the optimal strategy for problem (3.1) in the class $\bar{\Sigma}$.

In particular, the vector $\mu_{0} \in \Delta_{0}$ is non-random and such that $H_{0}\left(\mu_{0}\right) \leq H_{t}(v)$ for all $v=\left(v_{1}, \ldots, v_{n}\right) \in \mathbf{R}^{n}$ such that $\left(v_{1} \widetilde{Y}_{0} \widetilde{S}_{0,1}^{-1}, \ldots, v_{n} \widetilde{Y}_{0} \widetilde{S}_{0, n}^{-1}\right) \in \Delta_{0}$. 
Note that the assumption on the existence of the vectors $\mu_{t}$ is not restrictive when $U$ is convex. The choice of $\mu_{t}$ is unique in this case.

\section{Goal achieving with quadratic criterion}

Starting from now and up to the end of the paper, we assume that $\Delta_{t} \equiv \mathbf{R}^{n}$ and $U(x)=x^{2}$. Let $a_{0}=\mathbf{E} \xi_{1}, Q_{0}=\mathbf{E} \xi_{1} \xi_{1}^{\top}$

$$
a_{t} \triangleq \mathbf{E}\left\{\xi_{t+1} \mid \mathcal{F}_{t}\right\}, \quad Q_{t} \triangleq \mathbf{E}\left\{\xi_{t+1} \xi_{t+1}^{\top} \mid \mathcal{F}_{t}\right\}, \quad t=0,1,2 \ldots
$$

By Condition 2.2(ii), $Q_{t}>0$ a.s. for all $t$.

Theorem 3.2. Under the assumptions of Theorem 3.1 with $U(x)=x^{2}$,

$$
\mu_{t}=-Q_{t}^{-1} a_{t}
$$

i.e.,

$$
\gamma_{t, k}=-Q_{t}^{-1} a_{t}\left(\widetilde{X}_{t}-\kappa\right) \widetilde{S}_{t, k}^{-1}
$$

In addition,

$$
\mathbf{E}\left(\widetilde{X}_{T}-\kappa\right)^{2}=\alpha_{T}\left(X_{0}-\kappa\right)^{2}, \quad \mathbf{E} \widetilde{X}_{T}=\alpha_{T}\left(X_{0}-\kappa\right)+\kappa,
$$

where $\widetilde{X}_{T}$ is the corresponding discounted wealth, and where

$$
\alpha_{T} \triangleq \mathbf{E} \prod_{t=0}^{T-1}\left(1-a_{t}^{\top} Q_{t}^{-1} a_{t}\right) .
$$

Note that, in Theorem 3.2, $\mathbf{E} \tilde{X}_{T}=X_{0}+\left(X_{0}-\kappa\right)\left(\alpha_{T}-1\right)>X_{0}$ if $\alpha_{T}<1$.

Remark 3.2. It can be seen that the optimal strategies in Theorems 3.1 and 3.2 are myopic; they do not depend at time $t$ on distributions of $\xi_{t+2}, \xi_{t+3}, \ldots$, and it does not depend on $T$. It is an analog of Merton's type myopic strategy known for continuous time market.

Remark 3.3. In fact, the strategies in Theorems 3.1 and 3.2 are such that we do not need to know the entire conditional distribution of $\left(\xi_{t+1}, \rho_{t+1}\right)$ given $\theta_{1}, \ldots, \theta_{t}$ for calculation of the portfolio at time $t$ : we need only the $n$-dimensional vector $\mu_{t}=\mu_{t}\left(\theta_{1}, \ldots, \theta_{t}\right)$ defined by this distribution. It reminds the situation in continuous time setting where the so-called "market price of risk" process is sufficient to define the optimal strategy for $\log$ and power utility functions; see the discussion in [3]. This parameter $-\mu_{t}$ can be considered as an analog of the so-called "market price of risk" process defined for the continuous time market.

\section{Mean-VARiance optimal PORTfolio SELECtion}

Let $T \in \mathcal{T}$ be a given integer. Let $\alpha \triangleq \alpha_{T}$ be defined by (3.7).

Consider the following optimization problem:

$$
\text { Minimize } \operatorname{Var} \widetilde{X}_{T} \text { subject to } \mathbf{E} \widetilde{X}_{T} \geq c \text { over } \gamma . \in \bar{\Sigma} \text {. }
$$

We assume that Conditions 2.1-2.2 are satisfied. In addition, we assume from now that the following condition is satisfied.

Condition 4.1. There exists $t \in\{0,1, \ldots, T-1\}$ such that $\mathbf{P}\left(a_{t} \neq 0\right)>0$.

This last condition ensures that there exists a strategy such that $\mathbf{E} \tilde{X}_{T} \geq c$. 
Theorem 4.1. The unique optimal strategy for problem (4.1) in the class $\bar{\Sigma}$ is the strategy defined in Theorem 3.2 for the goal achieving problem with $U(x)=x^{2}$ and with the target value

$$
\widehat{\kappa}=\frac{c-X_{0} \alpha}{1-\alpha}
$$

Therefore, the optimal strategy $\left\{\left(\beta_{t}, \gamma_{t, 1}, \ldots, \gamma_{t, n}\right)\right\}$ obtained in Theorem 4.1 is such that

$$
\gamma_{t, k}=\mu_{t, k} \widetilde{Y}_{t} \widetilde{S}_{t, k}^{-1}, \quad \beta_{t}=\frac{X_{t}-\gamma_{t}^{\top} S_{t}}{B_{t}}, \quad t=0,1, \ldots, T-1,
$$

where $\widetilde{Y}_{t} \triangleq \widetilde{X}_{t}-\widehat{\kappa}, X_{t}$ is the corresponding wealth, $\widetilde{X}_{t}$ is the corresponding discounted wealth, $\mu_{t}=-Q_{t}^{-1} a_{t}$, and where $Q_{t}, a_{t}$, and $\alpha$, are defined by (3.4), (3.7).

Remark 4.1. Since $\alpha=\alpha_{T}$ depends on $\left(T,\left\{\left(\theta_{t}, S_{t}, \rho_{t}\right)\right\}_{t=1}^{T-1}\right)$, the optimal strategy in Theorem 4.1 is nonmyopic. However, the required information about the future distributions and terminal time is very limited: it is contained in this single scalar parameter $\alpha$. Strategies with similar features were introduced in [6] for continuous time model: the optimal strategy for multi-stock market was represented as a myopic one given that a single scalar parameter is known (an integral of the squared risk premium process). Some development of this approach was obtained in $[2,4]$.

Remark 4.2. Since the effectiveness of the forecast is usually decreasing in time, it is natural to accept that $a_{t}^{\top} Q_{t}^{-1} a_{t} \rightarrow 0$ as $t \rightarrow+\infty$. In that case, the value $\widehat{\kappa}$ is not very sensitive with respect to the changes in the choice of large $T$ or with respect to the errors in the estimates of the future distributions for large $t$.

\section{Robustness of the efficient frontier property}

The fact that the forecast is required for a scalar parameter reduces impact of a forecast error. It can be seen from the following corollary.

Corollary 4.1. Let a strategy be calculated as suggested in Theorem 4.1 with $\alpha$ replaced by some $\widetilde{\alpha} \in(0,1)$. (For instance, this $\widetilde{\alpha}$ can be calculated for another time horizon $\widetilde{T} \neq T$, or it can be calculated using some wrong prior distribution hypothesis.) Then this strategy belongs to $\bar{\Sigma}$ and it is on the efficient frontier. More precisely, this $\widehat{\alpha} \in(0,1)$ defines a new target value

$$
\widetilde{\kappa} \triangleq \frac{c-X_{0} \widetilde{\alpha}}{1-\widetilde{\alpha}}
$$

such that the following holds:

(i) This strategy is optimal for the goal achieving problem with this target value $\widetilde{k}$; and

(ii) This strategy is optimal for the mean variance problem

$$
\text { Minimize } \operatorname{Var} \tilde{X}_{T} \text { subject to } \mathbf{E} \tilde{X}_{T} \geq \widetilde{c} \text {, }
$$

where $\widetilde{c}$ is defined from the equation

$$
\frac{\widetilde{c}-X_{0} \alpha}{1-\alpha}=\widetilde{\kappa} .
$$


The features pointed out in Remarks 4.1, 4.2, and Corollary 4.1, reduce the impact of non-myopicness of the optimal strategy from Theorem 4.1.

\section{An example}

Let $\theta_{t}$ be a random process with values in $\mathcal{Z}$, and let $w_{t}$ be a random process in $\mathbf{R}^{m}$ independent from the process $\theta_{t}$ and such the random variables $w_{t}$ are mutually independent. Let a function $F: \mathcal{Z} \times \mathbf{R}^{m} \rightarrow \mathbf{R}^{n}$ be given. Our assumptions cover the market model with $\xi_{t}=F\left(\theta_{t-1}, w_{t}\right)$. Since the optimal strategy for the goal achieving problem is myopic, it does not require to know the distribution of the process $\theta_{t}$. For instance, if $\theta_{t}$ is a Markov process, than the strategy does not require its transition probabilities. The optimal strategy in Theorem 4.1 requires to calculate the target value $\widetilde{k}$ and this requires to calculate $\alpha$. In particular, it requires transition probabilities of $\theta_{t}$, if $\theta_{t}$ is a Markov process.

Remark 4.3. Condition 2.1(i) is not satisfied for a case when $\left\{\left(\xi_{t}, \rho_{t}\right)\right\}$ is a general Markov process. This is why the myopicness in the last example for a special Markov process does not contradict the conclusion of Hakannson [9] that serial correlations may prevent the optimal strategy to be myopic for $U(x)=\sqrt{x}$.

\section{On the criterions with the total wealth}

The results of $\mathrm{Li}$ and $\mathrm{Ng}$ [13] and Henrotte [10] covers the model with random bond prices and with the total wealth presented in optimality criterion instead of the discounted wealth used in our setting. It makes difference for the case of random bond prices. More precisely, we do not consider the problems

$$
\text { Minimize } \mathbf{E} U\left(X_{T}-\kappa\right)
$$

or

$$
\text { Minimize } \operatorname{Var} X_{T} \text { subject to } \mathbf{E} X_{T} \geq \text { const. }
$$

Formally, problems (4.3), (4.4) with random bond prices can be embedded into our model, if one takes $B_{t} \equiv B_{0}$ and $\Delta_{t}=\left\{v=\left(v_{1}, \ldots, v_{n}\right): \sum_{k=1}^{n} S_{t, k} v_{k}=X_{t}\right\}$, i.e., if the admissible strategies are such that $\sum_{k=1}^{n} \gamma_{t, k} S_{t, k} \equiv X_{t}$. In that case, the bond price process can be included into the model as a component of the vector process $S_{t}$ (for instance, as $S_{t, 1}$ ). In our notations for the goal-achieving problem, these constraints mean that $\left(X_{t}-\rho_{t} \kappa\right) \sum_{k=1}^{n} \mu_{t, k} \equiv X_{t}$. This approach requires minimization of $H_{t}(v)$ over the sets $\Delta_{t}$ that depend on $X_{t}$ defined by the past prices and past portfolio choices. We leave it for the future research.

\section{A. Appendix: Proofs}

Proof of Proposition 2.1. repeats the proof of Proposition 2.1 for the case $n=1$ from Dokuchaev [1], p. 17, and we omit it here.

Proof of Theorem 3.1. By (3.2), $\gamma_{t, k}=\mu_{t, k} \widetilde{Y}_{t} \widetilde{S}_{t, k}^{-1}$, and Proposition 2.1 implies that

$$
\widetilde{X}_{t+1}-\widetilde{X}_{t}=\widetilde{Y}_{t+1}-\widetilde{Y}_{t}=\gamma_{t}^{\top}\left[\widetilde{S}_{t+1}-\widetilde{S}_{t}\right]=\gamma_{t}^{\top} \widetilde{S}_{t} \xi_{t+1}=\mu_{t}^{\top} \xi_{t+1} \widetilde{Y}_{t}
$$

By (A.1),

It follows that

$$
\widetilde{Y}_{t}=\widetilde{Y}_{t-1}+\widetilde{Y}_{t-1} \mu_{t-1}^{\top} \xi_{t}, \quad t \geq 1
$$

$$
\widetilde{Y}_{T}=\widetilde{Y}_{0} \prod_{t=1}^{T}\left(1+\mu_{t-1}^{\top} \xi_{t}\right) .
$$

Let us show that $\gamma \in \bar{\Sigma}$. By Condition 2.2(ii), it follows that $\left|\mu_{t}\right| \leq c$ given $\theta_{1}, \ldots, \theta_{t}$, where $c=c\left(\theta_{1}, \ldots, \theta_{t}\right) \in$ $[0,+\infty)$. By Condition 2.2(i), it follows that $\mathbf{E}\left\{\left|\widetilde{Y}_{t}\right| \mid \theta_{1}, \ldots, \theta_{t}\right\}<+\infty, \mathbf{E}\left\{\left|\widetilde{X}_{t}\right| \mid \theta_{1}, \ldots, \theta_{t}\right\}<+\infty$ a.s., and, therefore, $\mathbf{E}\left\{\left|X_{t}\right| \mid \theta_{1}, \ldots, \theta_{t}\right\}<+\infty$ a.s. for $t \geq 0$. Hence the strategy (3.2) is admissible and $\gamma . \in \bar{\Sigma}$. 
Let us show that the strategy $\gamma$. is optimal in the class $\bar{\Sigma}$. Consider an arbitrary admissible strategy $\gamma^{\prime}=\left\{\left(\gamma_{t, 1}^{\prime}, \ldots, \gamma_{t, n}^{\prime}\right)\right\} \in \bar{\Sigma}$. Let $\widetilde{X}_{t}^{\prime}$ be the corresponding discounted wealth, $\widetilde{Y}_{t}^{\prime}=\widetilde{X}_{t}^{\prime}-\kappa$. Set

$$
\mu_{t, k}^{\prime} \triangleq\left\{\begin{array}{ll}
\gamma_{t, k}^{\prime} \widetilde{S}_{t, k}{\widetilde{Y_{t}^{\prime}}}^{-1}, & \widetilde{Y}_{t}^{\prime} \neq 0, \\
0, & \widetilde{Y}_{t}^{\prime}=0,
\end{array} \quad t \geq 0 .\right.
$$

Clearly, $\gamma_{t, k}^{\prime}=\mu_{t, k}^{\prime} \widetilde{Y}_{t}^{\prime} \widetilde{S}_{t, k}^{-1}$ a.s. for $t \geq 0$, and

$$
\begin{gathered}
\tilde{Y}_{t}^{\prime}=\tilde{Y}_{t-1}^{\prime}+\mu_{t-1}^{\prime} \xi_{t} \tilde{Y}_{t-1}^{\prime}, \quad t \geq 1, \\
\tilde{Y}_{T}^{\prime}=\widetilde{Y}_{0} \prod_{t=1}^{T}\left(1+\mu_{t-1}^{\prime}{ }^{\top} \xi_{t}\right) .
\end{gathered}
$$

Set $\Theta \triangleq\left(\theta_{1}, \ldots, \theta_{T-1}\right)$ for $T>1$. To cover the case when $T=1$, we assume that $\Theta=1$ if $T=1$; in that case, the conditional expectations $\mathbf{E}\{\cdot \mid \Theta\}$ below became unconditional expectations.

We have that

$$
\mathbf{E}\left\{U\left(\widetilde{Y}_{T}^{\prime}\right) \mid \Theta\right\}=U\left(\widetilde{Y}_{0}\right) \mathbf{E}\left\{\prod_{t=1}^{T} U\left(1+\mu_{t-1}^{\prime}{ }^{\top} \xi_{t}\right) \mid \Theta\right\}
$$

Set

$$
V_{t} \triangleq U\left(1+\mu_{t-1}^{\top} \xi_{t}\right), \quad V_{t}^{\prime} \triangleq U\left(1+\mu_{t-1}^{\prime}{ }^{\top} \xi_{t}\right)
$$

Set

$$
W_{t} \triangleq\left(\rho_{1}, \xi_{1}, \ldots, \rho_{t}, \xi_{t}, \Theta\right), \quad t>0, \quad W_{0}=\Theta .
$$

Let $\mathbf{E}_{W_{t}}$ denotes the conditional expectation $\mathbf{E}\left\{\cdot \mid W_{t}\right\}$, and let $\mathbf{E}_{W_{0}}=\mathbf{E}$. We introduce the following sequences of random variables:

$$
\psi_{T} \triangleq \mathbf{E}_{W_{T-1}} V_{T}, \quad \psi_{t} \triangleq \mathbf{E}_{W_{t-1}}\left[V_{t} \psi_{t+1}\right], \quad t=T-1, \ldots, 1,
$$

and

$$
\psi_{T}^{\prime} \triangleq \mathbf{E}_{W_{T-1}} V_{T}^{\prime}, \quad \psi_{t}^{\prime} \triangleq \mathbf{E}_{W_{t-1}}\left[V_{t}^{\prime} \psi_{t+1}\right], \quad t=T-1, \ldots, 1
$$

By the definitions,

$$
\mathbf{E}\left\{U\left(\widetilde{Y}_{T}\right) \mid \Theta\right\}=U\left(\widetilde{Y}_{0}\right) \mathbf{E}\left\{\psi_{1} \mid \Theta\right\}, \quad \mathbf{E}\left\{U\left(\widetilde{Y}_{T}^{\prime}\right) \mid \Theta\right\}=U\left(\widetilde{Y}_{0}\right) \mathbf{E}\left\{\psi_{1}^{\prime} \mid \Theta\right\} .
$$

By Condition 2.1(i), the vectors $\left(\xi_{t}, \rho_{t}\right), t \geq 1$, are conditionally independent given $\Theta$. By the definitions, $\mu_{t}$ are non-random for any $t$ in the conditional probability space given $\Theta$. It follows that $\psi_{t}$ are non-random for any $t$ in the conditional probability space given $\Theta$.

By (3.3),

$$
\mathbf{E}_{W_{T-1}} V_{T} \leq \mathbf{E}_{W_{T-1}} \bar{V}_{T}^{\prime} \quad \text { a.s. }
$$

Hence

$$
\psi_{T} \leq \psi_{T}^{\prime} \quad \text { a.s. }
$$


By (3.3) again, it follows that

$$
\mathbf{E}_{W_{t-1}} V_{t} \leq \mathbf{E}_{W_{t-1}} V_{t}^{\prime} \quad \text { a.s. }
$$

Let $t \in\{2, \ldots, T\}$. Let us show that if

$$
\psi_{t} \leq \psi_{t}^{\prime} \quad \text { a.s. }
$$

then

$$
\psi_{t-1} \leq \psi_{t-1}^{\prime} \quad \text { a.s. }
$$

Let (A.6) be satisfied. Remind that $\psi_{t}$ is non-random on the conditional probability space given $\Theta$. By (A.5), it follows that

$$
\psi_{t-1}=\mathbf{E}_{W_{t-1}}\left[V_{t} \psi_{t}\right] \leq \mathbf{E}_{W_{t-1}}\left[V_{t}^{\prime} \psi_{t}\right] \leq \mathbf{E}_{W_{t-1}}\left[V_{t}^{\prime} \psi_{t}^{\prime}\right]=\psi_{t-1}^{\prime} \quad \text { a.s. }
$$

Hence (A.7) follows from (A.6). Therefore, it can be concluded that $\psi_{1} \leq \psi_{1}^{\prime}$ a.s. By (A.4), it follows that $\mathbf{E}\left\{U\left(\widetilde{Y}_{T}\right) \mid \Theta\right\} \leq \mathbf{E}\left\{U\left(\widetilde{Y}_{T}^{\prime}\right) \mid \Theta\right\}$ a.s. Hence $\mathbf{E}\left\{U\left(\widetilde{Y}_{T}\right) \mid \Theta\right\} \geq \mathbf{E}\left\{U\left(\widetilde{Y}_{T}^{\prime}\right) \mid \Theta\right\}$ a.s. This completes the proof of Theorem 3.1.

Proof of Theorem 3.2. By Condition 2.1(i), we have that

$$
a_{t} \triangleq \mathbf{E}\left\{\xi_{t+1} \mid \theta_{1}, \ldots, \theta_{t}\right\}, \quad Q_{t} \triangleq \mathbf{E}\left\{\xi_{t+1} \xi_{t+1}^{\top} \mid \theta_{1}, \ldots, \theta_{t}\right\}, \quad t \geq 0 .
$$

Further,

$$
\begin{aligned}
H_{t}(v) & =\mathbf{E}\left\{U\left(1+v^{\top} \xi_{t+1}\right) \mid \mathcal{F}_{t}\right\}=\mathbf{E}\left\{\left(1+v^{\top} \xi_{t+1}\right)^{2} \mid \theta_{1}, \ldots, \theta_{t}\right\} \\
& =1+2 v^{\top} a_{t}+v^{\top} Q_{t} v=\left(v+Q_{t}^{-1} a_{t}\right)^{\top} Q_{t}\left(v+Q_{t}^{-1} a_{t}\right)+1-a_{t}^{\top} Q_{t}^{-1} a_{t} .
\end{aligned}
$$

Hence $\mu_{t}=-Q_{t}^{-1} a_{t}$ is the unique vector that solves (3.3) for $U(x)=x^{2}$. For this $\mu_{t}$,

$$
\mathbf{E} \prod_{t=0}^{T-1}\left(1+\mu_{t}^{\top} \xi_{t+1}\right)^{2}=\mathbf{E} \mathbf{E}\left\{\prod_{t=0}^{T-1}\left(1+\mu_{t}^{\top} \xi_{t+1}\right)^{2} \mid \Theta\right\}=\mathbf{E} \prod_{t=0}^{T-1}\left(1-a_{t}^{\top} Q_{t} a_{t}\right)=\alpha_{T} .
$$

By (A.2), it follows that, for this $\mu_{t}$,

$$
\begin{aligned}
\mathbf{E}\left(\widetilde{X}_{T}-\kappa\right)^{2} & \left.=\mathbf{E E}\left(\widetilde{X}_{T}-\kappa\right)^{2} \mid \Theta\right\}=\left(X_{0}-\kappa\right)^{2} \mathbf{E} \prod_{t=0}^{T-1} H_{t}\left(\mu_{t}\right) \\
& =\left(X_{0}-\kappa\right)^{2} \mathbf{E} \prod_{t=0}^{T-1}\left(1-a_{t}^{\top} Q_{t}^{-1} a_{t}\right)=\left(X_{0}-\kappa\right)^{2} \alpha_{T} .
\end{aligned}
$$

We used for the second equality here that $a_{t}$ and $Q_{t}$ are non-random on the conditional probability space given $\Theta$. Similarly,

$$
\left.\mathbf{E}\left(\widetilde{X}_{T}-\kappa\right)=\mathbf{E} \mathbf{E}\left(\widetilde{X}_{T}-\kappa\right) \mid \Theta\right\}=\left(X_{0}-\kappa\right) \mathbf{E} \prod_{t=0}^{T-1}\left(1-a_{t}^{\top} Q_{t}^{-1} a_{t}\right)=\left(X_{0}-\kappa\right) \alpha_{T}
$$

This completes the proof of Theorem 3.2.

Proof of Theorem 4.1. Let mappings $\Phi_{i}: \bar{\Sigma} \rightarrow \mathbf{R}$ be defined as $\Phi_{0}(\gamma)=.\mathbf{E} \widetilde{X}_{T}^{2}, \Phi_{1}(\gamma)=.\mathbf{E} \widetilde{X}_{T}$, where $\widetilde{X}_{T}$ is the corresponding discounted wealth. 
Proposition A.1. Problem (4.1) has the same set of solutions as any of the following problems of minimization over $\gamma \cdot \in \bar{\Sigma}$ :

(i)

$$
\text { Minimize } \operatorname{Var} \widetilde{X}_{T} \text { subject to } \mathbf{E} \widetilde{X}_{T}=c \text {. }
$$

(ii)

$$
\text { Minimize } \mathbf{E} \tilde{X}_{T}^{2} \text { subject to } \mathbf{E} \tilde{X}_{T}=c \text {. }
$$

(iii)

$$
\text { Minimize } \mathbf{E} \widetilde{X}_{T}^{2} \text { subject to } \mathbf{E} \widetilde{X}_{T} \geq c \text {. }
$$

Proof of Proposition A.1. Since $\operatorname{Var} \widetilde{X}_{T}=\mathbf{E} \widetilde{X}_{T}^{2}-\left(\mathbf{E} \widetilde{X}_{T}\right)^{2}$, we have that problems (A.9) and (A.10) are equivalent, i.e., they have the same sets of the solutions. Further, let $J \triangleq \inf \mathbf{E} \widetilde{X}_{T}^{2}$ for problem (A.10). It follows that there exists a sequence $\left\{\gamma_{.}^{k}\right\}_{k=1}^{+\infty} \subset \bar{\Sigma}$ such that $\Phi_{0}\left(\gamma_{.}^{k}\right) \rightarrow J$ as $k \rightarrow+\infty$ and $\Phi_{1}\left(\gamma_{.}^{k}\right)=h_{k} c$, where $h_{k} \geq 1$. Set $\widehat{\gamma}^{k} \triangleq h_{k}^{-1} \gamma_{.}^{k}$. The kind of the functions $\Phi_{i}$ is such that $\Phi_{0}\left(\widehat{\gamma}_{.}^{k}\right)=h_{k}^{-2} \Phi_{0}\left(\gamma_{.}^{k}\right) \leq \Phi_{0}\left(\widehat{\gamma}^{k}\right)$ and $\Phi_{1}\left(\widehat{\gamma}^{k}\right)=c$. It follows that the wider condition in problem (A.11) does not improve the performance of optimal strategies comparing with problem (A.10). Similarly, it can be shown that problem (4.1) is equivalent to problem (A.9). This completes the proof.

Let us continue the proof of Theorem 4.1. It follows from Proposition A.1 that it suffices to solve problem (A.11).

Let us show first that the strategy presented is optimal.

Let us introduce the Lagrangian for the problem (A.11)

$$
L(\gamma ., q) \triangleq \Phi_{0}(\gamma \cdot)+q\left(c-\Phi_{1}(\gamma \cdot)\right)=\mathbf{E} \widetilde{X}_{T}^{2}+q\left(c-\mathbf{E} \widetilde{X}_{T}\right) .
$$

It can rewritten as

$$
L(\gamma,, q)=\mathbf{E}\left(\widetilde{X}_{T}-\frac{q}{2}\right)^{2}+q c-\frac{q^{2}}{4} .
$$

Let

$$
\widehat{q}=\frac{2\left(c-X_{0} \alpha\right)}{1-\alpha}
$$

Consider now the problem

$$
\text { Minimize } L(\gamma ., \widehat{q})=\mathbf{E}\left(\widetilde{X}_{T}-\frac{\widehat{q}}{2}\right)^{2}+\widehat{q} c-\frac{\widehat{q}^{2}}{4} \quad \text { over } \gamma \cdot \in \bar{\Sigma} .
$$

Theorem 3.2 applied for $\widehat{\kappa}=\widehat{q} / 2$ and $U(x)=x^{2}$ gives the unique optimal solution $\widehat{\gamma}$. of problem (A.12).

Further, it follows from (3.6) that the corresponding discounted wealth is such that

$$
\begin{aligned}
\mathbf{E} \widetilde{X}_{T} & =\alpha\left(X_{0}-\frac{\widehat{q}}{2}\right)+\frac{\widehat{q}}{2}=\alpha\left(X_{0}-\frac{2\left(c-X_{0} \alpha\right)}{1-\alpha}\right)+\frac{2\left(c-X_{0} \alpha\right)}{1-\alpha} \\
& =\frac{1}{1-\alpha}\left((1-\alpha) \alpha X_{0}-\alpha c+\alpha^{2} X_{0}+c-\alpha X_{0}\right)=c .
\end{aligned}
$$


Set $\bar{\Sigma}_{0}=\left\{\gamma \cdot \in \bar{\Sigma}: \mathbf{E} \widetilde{X}_{T} \geq c\right\}$. Let $\gamma \cdot \in \bar{\Sigma}_{0}$, then

$$
\Phi_{0}(\gamma .)-\Phi_{0}(\widehat{\gamma} .) \geq L(\gamma ., \widehat{q})-L(\widehat{\gamma},, \widehat{q}) \geq 0
$$

Therefore, the strategy $\gamma$. is optimal for the problem (4.1).

Let us show that the optimal strategy is unique. Note that Condition 4.1 ensures that the following Slater condition is satisfied:

$$
\exists \gamma . \in \bar{\Sigma}: \mathbf{E} \widetilde{X}_{T}>c .
$$

The following proposition follows from classical Lagrange Duality Theorem (see, e.g., [16], Chap. 8.6, p. 224).

\section{Proposition A.2.}

(i) The following relation holds:

$$
\inf _{\gamma \cdot \in \bar{\Sigma}_{0}} \widetilde{X}_{T}^{2}=\inf _{\gamma \cdot \in \bar{\Sigma}} \sup _{q \geq 0} L(\gamma \cdot, q)=\sup _{q \geq 0} \inf _{\gamma \cdot \in \bar{\Sigma}} L(\gamma,, q) .
$$

The supremum on the right-hand side of (A.14) is achievable for a finite $\widehat{q} \geq 0$.

(ii) If the infimum on the left of (A.14) is achieved for some $\gamma . \in \bar{\Sigma}$ (i.e., it is the solution of the minimization problems with constraints (4.1)), then

$$
\widehat{q}\left(\mathbf{E} \widetilde{X}_{T}-c\right)=0 .
$$

The pair $(\gamma ., \widehat{q})$ is the solution of the problem $\sup _{q \geq 0} \inf _{\gamma, \in \bar{\Sigma}} L(\gamma ., q)$, and $\gamma$. is the solution of the problem $\inf _{\gamma, \in \bar{\Sigma}} L(\gamma ., \widehat{q})$.

We have that $\gamma .=\widehat{\gamma}$. $(q)$ defined by Theorem 3.2 with $U(x)=x^{2}$ and $\kappa=q / 2$ is the unique solution of the problem $\inf _{\gamma, \in \bar{\Sigma}} L(\gamma, q)$.

By Theorem 3.2, we have

$$
L(\widehat{\gamma} \cdot(q), q)=\left(X_{0}-\frac{q}{2}\right)^{2} \mathbf{E} \prod_{t=0}^{T-1}\left(1+\mu_{t}^{\top} \xi_{t+1}\right)^{2}+q c-\frac{q^{2}}{4}=\left(X_{0}-\frac{q}{2}\right)^{2} \alpha+q c-\frac{q^{2}}{4} .
$$

We used (A.8) here.

Clearly, $\alpha=\alpha_{T} \in(0,1)$, and the problem

$$
\text { Maximize } L(\widehat{\gamma} \cdot(q), q)=\left(X_{0}-\frac{q}{2}\right)^{2} \alpha+q c-\frac{q^{2}}{4} \quad \text { over } q>0
$$

has the unique solution $\widehat{q}$. Therefore, $\widehat{q}$ is the unique solution of the problem $\sup _{q \geq 0} L(\widehat{\gamma} \cdot(q), q)$, and the pair $(\widehat{\gamma} .(\widehat{q}), \widehat{q})$ is the unique solution of the ${\operatorname{problem~} \sup _{q \geq 0} \inf }_{\gamma, \in \bar{\Sigma}} L(\gamma ., q)$. Then the uniqueness of optimal strategy follows from Proposition A.2(ii). This completes the proof of Theorem 4.1.

Proof of Corollary 4.1. The choice of $\alpha$ is used for the calculation of the target value only. Therefore, the pair $(\widetilde{\alpha}, c)$ gives the same strategy as the pair $(\alpha, \widetilde{c})$. This completes the proof.

Acknowledgements. This work was supported by NSERC grant of Canada 341796-2008 to the author. 


\section{REFERENCES}

[1] N.G. Dokuchaev, Dynamic portfolio strategies: quantitative methods and empirical rules for incomplete information. Kluwer, Boston (2002).

[2] N. Dokuchaev, Saddle points for maximin investment problems with observable but non-predictable parameters: solution via heat equation. IMA J. Management Mathematics 17 (2006) 257-276.

[3] N. Dokuchaev, Discrete time market with serial correlations and optimal myopic strategies. European J. Oper. Res. 177 (2007) 1090-1104.

[4] N. Dokuchaev, Maximin investment problems for discounted and total wealth. IMA J. Management Mathematics 19 (2008) 63-74.

[5] N Dokuchaev, Optimality of myopic strategies for multi-stock discrete time market with management costs. European J. Oper. Res. (to appear).

[6] N.G. Dokuchaev and U. Haussmann, Optimal portfolio selection and compression in an incomplete market. Quantitative Finance 1 (2001) 336-345.

[7] E. Dynkin and I. Evstigneev, Regular conditional expectations of correspondences. Theory Probab. Appl. 21 (1976) 325-338.

[8] D. Feldman, Incomplete information equilibria: separation theorem and other myths. Ann. Oper. Res. 151 (2007) $119-149$.

[9] N.H. Hakansson, On optimal myopic portfolio policies, with and without serial correlation of yields. J. Bus. 44 (1971) 324-334.

[10] P. Henrotte, Dynamic mean variance analysis. Working paper, SSRN: http://ssrn. com/abstract=323397 (2002).

[11] C. Hipp and M. Taksar, Hedging general claims and optimal control. Working paper (2000).

[12] H. Leland, Dynamic Portfolio Theory. Ph.D. Thesis, Harvard University, USA (1968).

[13] D. Li and W.L. Ng, Optimal portfolio selection: multi-period mean-variance optimization. Math. Finance 10 (2000) $387-406$.

[14] A. Lim, Quadratic hedging and mean-variance portfolio selection with random parameters in an incomplete market. Math. Oper. Res. 29 (2004) 132-161.

[15] A. Lim and X.Y. Zhou, Mean-variance portfolio selection with random parameters in a complete market. Math. Oper. Res. 27 (2002) 101-120.

[16] D.G. Luenberger, Optimization by Vector Space Methods. John Wiley, New York (1968).

[17] H.M. Markowitz, Portfolio Selection: Efficient Diversification of Investment. New York: John Wiley \& Sons (1959).

[18] R. Merton, Lifetime portfolio selection under uncertainty: the continuous-time case. Rev. Economics Statistics 51 (1969) $247-257$.

[19] J. Mossin, Optimal multi-period portfolio policies. J. Business 41 (1968) 215-229.

[20] S.R. Pliska, Introduction to mathematical finance: discrete time models. Blackwell Publishers (1997).

[21] M. Schweizer, Variance-optimal hedging in discrete time. Math. Oper. Res. 20 (1995) 1-32. 